\title{
The effects of green coffee extract supplementation on glycemic indices and lipid profile in adults: a systematic review and dose-response meta-analysis of clinical trials
}

Omid Asbaghi ${ }^{1}$, Mehdi Sadeghian ${ }^{2}$, Morteza Nasiri ${ }^{3,4}$, Mahmoud Khodadost ${ }^{5,6}$, Azad Shokri $^{7}$, Bahman Panahande ${ }^{8}$, Aliyar Pirouzi ${ }^{9}$ and Omid Sadeghi ${ }^{10,11^{*}}$

\begin{abstract}
Background: The role of coffee consumption in the risk of cardiovascular diseases has been debated for many years. The current study aimed to summarize earlier evidence on the effects of green coffee extract (GCE) supplementation on glycemic indices and lipid profile.

Methods: We searched available online databases for relevant clinical trials published up to October 2019. All clinical trials investigating the effect of GCE supplementation, compared with a control group, on fasting blood glucose (FBG), serum insulin, total cholesterol (TC), triglyceride (TG), low-density lipoprotein (LDL), and high-density lipoprotein (HDL) were included. Overall, 14 clinical trials with a total sample size of 766 participants were included in the current meta-analysis.
\end{abstract}

Results: We found a significant reducing effect of GCE supplementation on FBG (weighted mean difference (WMD): $-2.35,95 \% \mathrm{Cl}:-3.78,-0.92 \mathrm{mg} / \mathrm{dL}, P=0.001)$ and serum insulin (WMD: $-0.63,95 \% \mathrm{Cl}:-1.11,-0.15 \mu \mathrm{U} / \mathrm{L}, P=0.01$ ). With regard to lipid profile, we observed a significant reduction only in serum levels of TC following GCE supplementation in the overall meta-analysis (WMD: $-4.51,95 \% \mathrm{Cl}$ : $-8.39,-0.64, P=0.02)$. However, subgroup analysis showed a significant reduction in serum TG in studies enrolled both genders. Also, such a significant reduction was seen in serum levels of $L D L$ and $H D L$ when the analyses confined to studies with intervention duration of $\geq 8$ weeks and those included female subjects. In the non-linear dose-response analyses, we found that the effects of chlorogenic acid (CGA) dosage, the main polyphenol in GCE, on FBG, TG and HDL were in the nonlinear fashions.

\footnotetext{
* Correspondence: omidsadeghi69@yahoo.com

${ }^{10}$ Students' Scientific Research Center, Tehran University of Medical Sciences, Tehran, Iran

${ }^{11}$ Department of Community Nutrition, School of Nutritional Sciences and Dietetics, Tehran University of Medical Sciences, Tehran, Iran

Full list of author information is available at the end of the article
}

C C The Author(s). 2020 Open Access This article is licensed under a Creative Commons Attribution 4.0 International License, which permits use, sharing, adaptation, distribution and reproduction in any medium or format, as long as you give appropriate credit to the original author(s) and the source, provide a link to the Creative Commons licence, and indicate if changes were made. The images or other third party material in this article are included in the article's Creative Commons licence, unless indicated otherwise in a credit line to the material. If material is not included in the article's Creative Commons licence and your intended use is not permitted by statutory regulation or exceeds the permitted use, you will need to obtain permission directly from the copyright holder. To view a copy of this licence, visit http://creativecommons.org/licenses/by/4.0/ The Creative Commons Public Domain Dedication waiver (http://creativecommons.org/publicdomain/zero/1.0/) applies to the data made available in this article, unless otherwise stated in a credit line to the data. 
(Continued from previous page)

Conclusion: In conclusion, we found that GCE supplementation improved FBG and serum levels of insulin and TC. Also, there was a significant improvement in other markers of lipid profile in some subgroups of clinical trials.

Keywords: Chlorogenic acid, Green coffee, Lipid profile, Glycemic indices

\section{Background}

Cardiovascular diseases (CVDs) are the number one cause of death worldwide [1]. Dyslipidemia and impaired glucose tolerance (IGT) are among the primary risk factors for the development and progression of CVDs and type 2 diabetes [2, 3]. Reversal of these risk factors leads to a considerable reduction in the risk of these chronic diseases [4-7]. Although current guideline recommends dietary regulations as the first-line therapy for dyslipidemia and glucose disturbance, only a modest amelioration has been achieved using these methods [8].

Coffee is widely consumed in the world containing a range of phytochemicals. Previous studies have shown beneficial effects of coffee consumption on several health conditions including metabolic syndrome, type 2 diabetes, and vascular function $[9,10]$. The most commonly found phytochemicals in the coffee are phenolic compounds, primarily chlorogenic acid (CGA) [11]. CGA is the ester of caffeic acid with quinic acid that belongs to the family of hydroxycinnamic acid [12]. Anti-lipidemic and anti-diabetic properties of CGA have been demonstrated in animal studies [13-15]. However, findings from human clinical trials are not consistent [16-29]. Green coffee extract (GCE) and its CGA showed hypolipidemic effects on serum levels of triglyceride (TG) and total cholesterol (TC) in patients with IGT [29] and those with non-alcoholic fatty liver disease [26]; however, the effects on circulating levels of high-density lipoprotein (HDL) and low-density lipoprotein (LDL) were inconsistent. Some studies showed a significant increase in serum HDL following GCE intake [17, 19], while others could not find any significant results $[20,21,24]$. Considering insulin resistance, fasting blood glucose (FBG) or serum insulin significantly reduced following GCE administration in some studies $[18,24,29]$, but no significant changes were observed in some others $[23,27]$.

A previous meta-analysis of randomized controlled trials (RCTs) found increased levels of serum TC, LDL, and TG following coffee intake [30]; however, to our knowledge, there is no study summarizing available findings on the effects of green coffee consumption on glycemic indices and lipid profile. Therefore, we aimed to conduct a systematic review and meta-analysis to summarize current evidence on the effects of GCE supplementation on glycemic and lipid profiles in adults.

\section{Methods}

This study was performed based on the PRISMA (Preferred Reporting Items for Systematic Reviews and Meta-Analyses) protocol for reporting systematic reviews and meta-analyses [31].

\section{Search strategy}

A comprehensive literature search was performed using online databases of PubMed, Scopus, Web of Science and Google Scholar up to October 2019. The aim of the search was to identify clinical trials that investigated the effects of GCE supplementation on glycemic indices and lipid profile in adults. The following keywords were used in the search strategy: ("Green coffee" OR "Green coffee extract" OR "Green coffee bean extract" OR "Chlorogenic acid" OR "Chlorogenic" OR "GCR" OR "CGA") AND (Triglyceride OR Triacylglycerol OR TG OR cholesterol OR lipoprotein OR "very low density lipoprotein" OR VLDL OR "low density lipoprotein" OR LDL OR "high density lipoprotein" OR HDL OR "lipid profile" OR "fasting blood sugar" OR glucose OR insulin OR " glycosylated hemoglobin" OR HbA1c OR FBS OR FBG). No restriction was considered for the time and language of publications. We conducted a manual search in the reference lists of the relevant studies to avoid missing any eligible publication. Unpublished studies were not considered.

\section{Inclusion criteria}

We included eligible studies that met the following criteria: 1) placebo-controlled clinical trials 2) those that performed on adult subjects ( $\geq 18$ years old), 3 ) studies that administered green coffee extract in the forms of supplement or powder added to foods or beverages, 4) those that did intervention for at least 2 weeks, and 5) controlled trials that reported mean changes and SDs of glycemic indices or lipid profile throughout the trial for both the intervention and control groups or presented required information for calculation of those effect sizes. If more than one article was found for one dataset, the more complete one was selected. Clinical trials with an additional arm were considered as 2 separate studies.

\section{Exclusion criteria}

In the current meta-analysis, we excluded:1) in vitro and animal studies, 2) studies with a cohort, cross-sectional, 
and case-control design, 3) review articles, 3) trials without a placebo or control group.

\section{Data extraction}

The following information was extracted from each eligible clinical trial by two independent investigators: name of the first author, publication year, individuals' characteristics (mean age and sex), design, sample size (control and intervention groups), type of intervention, dosage of GCE and CGA, duration of intervention, and mean changes and SDs of outcome variables throughout the trial for the intervention and control groups. When data for glycemic or lipid measures were reported in different units, we converted them to the most frequently used unit.

\section{Risk of bias assessment}

We used the Cochrane quality assessment tool to assess the risk of bias for each study included in the current meta-analysis [32]. This tool contained seven domains including random sequence generation, allocation concealment, reporting bias, performance bias, detection bias, attrition bias, and other sources of bias. Each domain was given a "high risk" score if RCT comprised methodological defects that may have distorted the results, a "low risk" score if the defect was considered ineffectual and an "unclear risk" score if the information was not sufficient to determine the impact. If the trial had "low risk" for all domains, it was labeled as a highquality study with a totally low risk of bias. The risk of bias assessment was done independently by two reviewers.

\section{Statistical analysis}

Mean differences in changes of the outcome variables (FBG, insulin, TG, TC, LDL, and HDL), comparing GCE and control groups, were used to obtain the overall effect sizes. When mean changes were not reported, we computed them by considering changes in each outcome variable during the intervention. If outcome variables (FBG, TG, TC, LDL, and HDL) were reported in $\mathrm{mmol} / \mathrm{L}$, we converted them to $\mathrm{mg} / \mathrm{dl}$ through available suitable formulas. We also converted standard errors (SEs), 95\% confidence intervals (CIs), and interquartile ranges (IQRs) to SDs using relevant formulas [33-35]. To obtain the overall effect sizes, we applied a random-effects model taking between-study variations into account. Heterogeneity was determined by the $\mathrm{I}^{2}$ statistic and Cochrane's Q test. $\mathrm{I}^{2}$ value $>50 \%$ or $P<0.05$ for the $\mathrm{Q}$-test was considered as significant between-study heterogeneity [36, 37]. To find probable sources of heterogeneity, subgroup analyses were performed according to the predefined criteria including gender (both/male/ female), length of intervention $(\geq 8 /<8$ weeks), baseline levels of glycemic and lipid measures (abnormal/normal levels) and participants' compliance (acceptable/ non-acceptable or unclear). To determine the nonlinear potential effects of CGA dosage $(\mathrm{mg} / \mathrm{d})$ on glycemic and lipid indices, fractional polynomial modeling was executed. Due to the lack of information on the dosage of GCE in some included studies, we decided to perform non-linear dose-response analysis for CGA dosage. Sensitivity analysis was used to explore the extent to which inferences might depend on a particular study. The possibility of publication bias was evaluated by the formal test of Begg. The metaanalysis was carried out by the use of the Stata, version 11.2 (StataCorp). $P$ value $<0.05$ was considered as significant level.

\section{Results}

After the initial search, a total of 1571 studies were identified. After removing duplicate publications, 976 articles remained, out of which 958 studies were identified as unrelated when screening for title and abstract. After assessing the full text of remained articles, we excluded one study in which the effects of GCE in combination with olive leaf and beetroot were assessed [38]. We also excluded a study by Salamat et al. [39] that reported data only for oxidized LDL, not the natural type. Two studies that were quasi-experimental with no control group were excluded as well [40, 41]. Two RCTs were conducted on a similar dataset; however, due to assessing different outcome variables, both of them were included $[21,25]$. Finally, 14 studies remained for the current systematic review and meta-analysis [16-29], out of which 11 studies presented data for FBG [16, 18, 19, 22-29], 7 studies for serum concentrations of insulin $[18,19,24-$ 26, 29], 13 trials for serum concentrations of TC [16-24, 26-29], and 12 trials for serum concentrations of TG, LDL, and HDL [17-24, 26-29]. Data on other glycemic indices including glycosylated hemoglobin A1c (HbA1c) $(n=3)$ and homeostatic model assessment for insulin resistance (HOMA-IR) $(n=2)$ were not sufficient for the meta-analysis. Flow diagram of study selection is presented in Supplemental Figure 1.

\section{Findings from the systematic review}

Characteristics of the 14 clinical trials included in the current systematic review are illustrated in Table 1. The total sample size of the selected studies was 766 adult participants including 380 subjects in the GCE group and 386 subjects in the control group. Studies were published from 2004 to 2019 . Out of 14 included studies, 3 were performed in western countries $[21,25,29]$ and others were conducted in Asia [16-20, 22-24, 26-28]. The dosage of GCE among the included studies was 


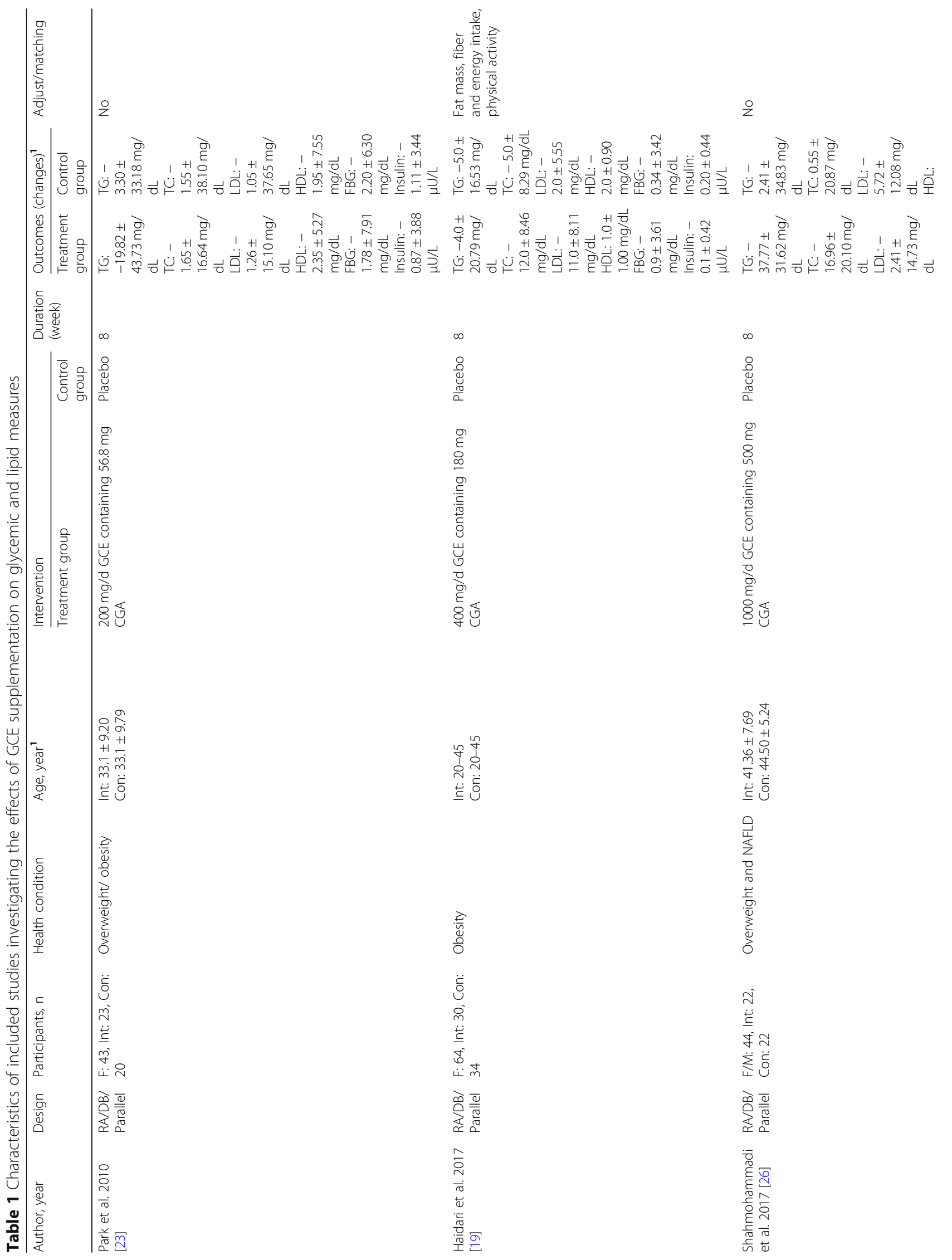




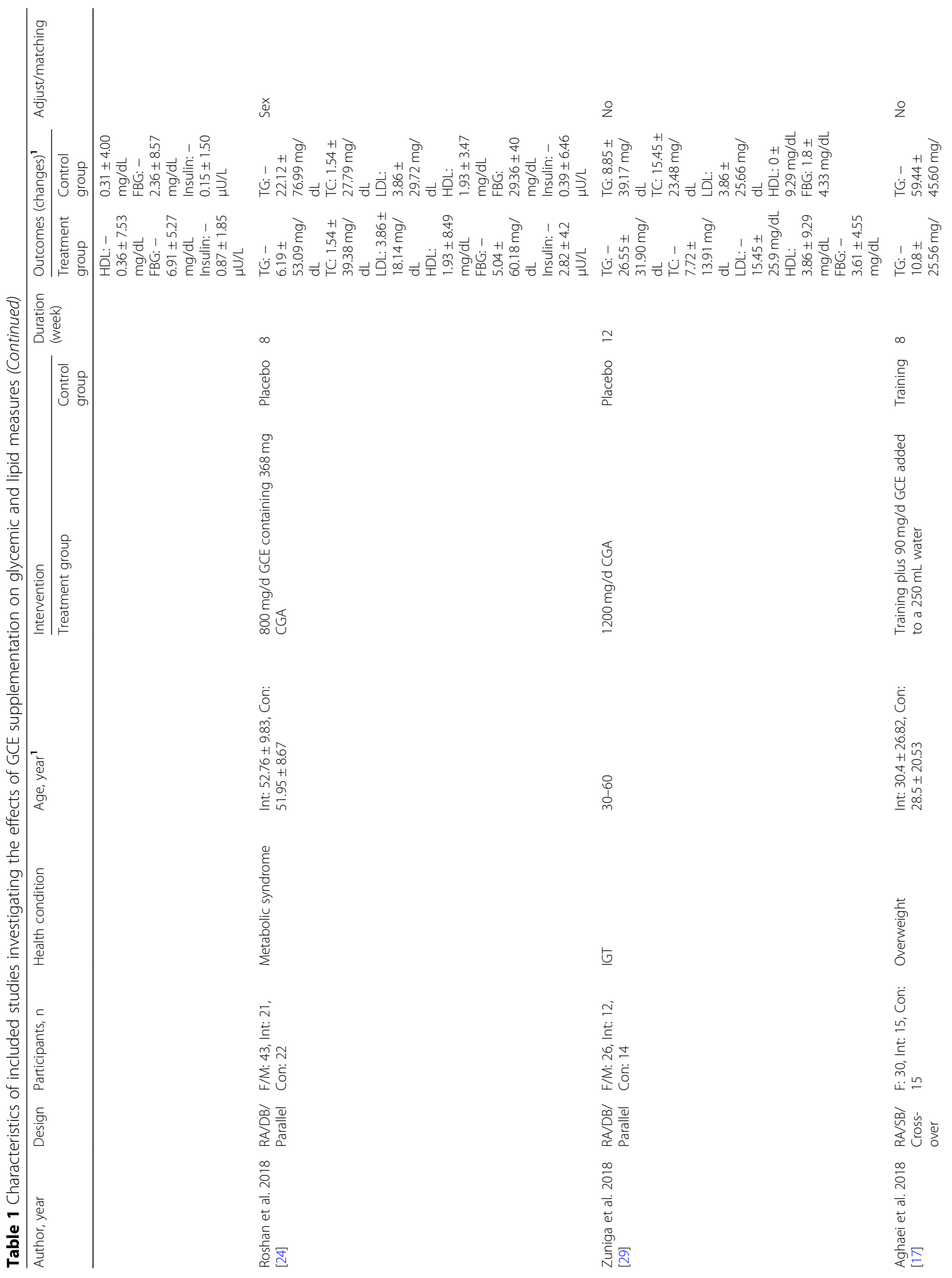




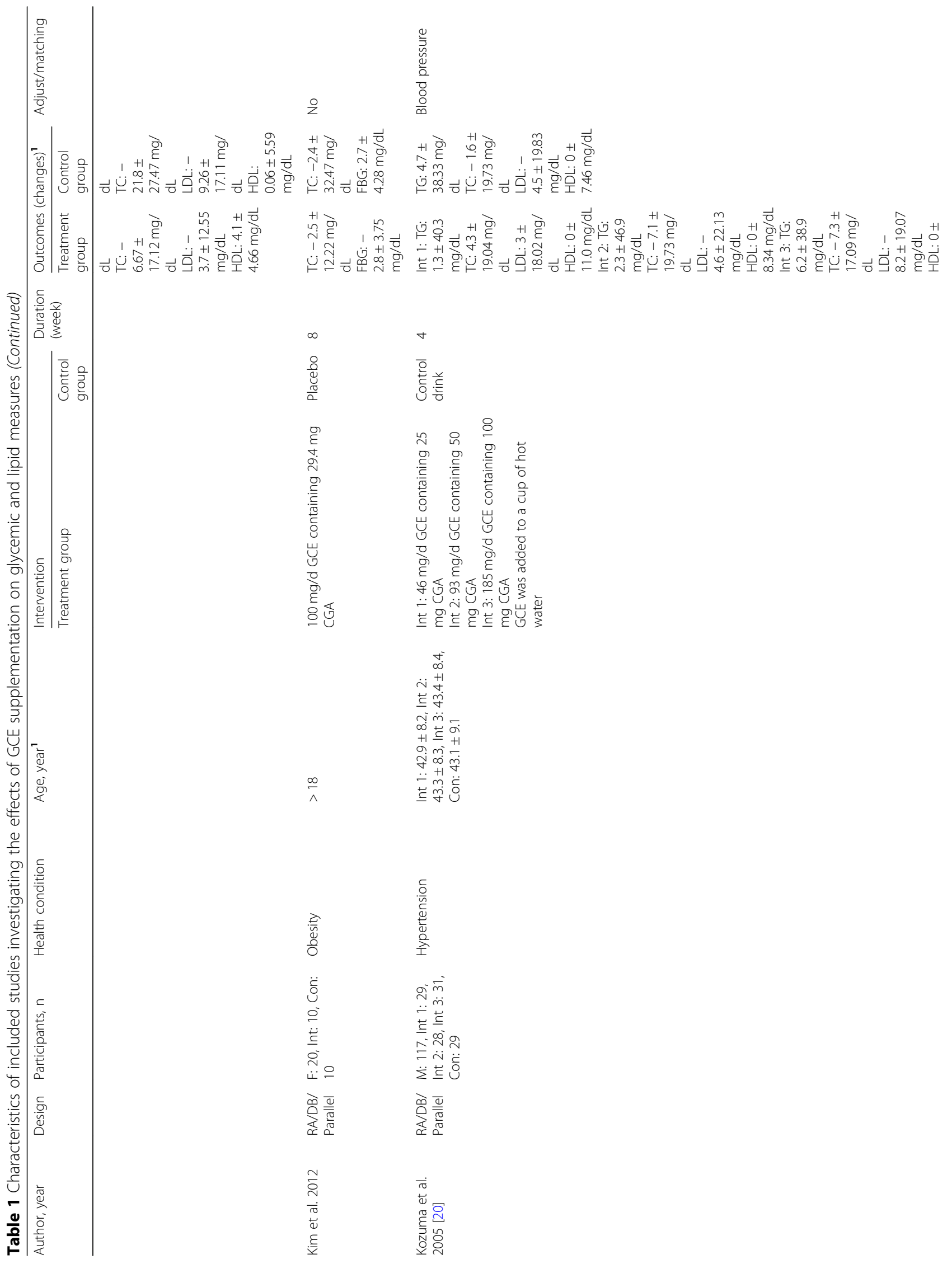




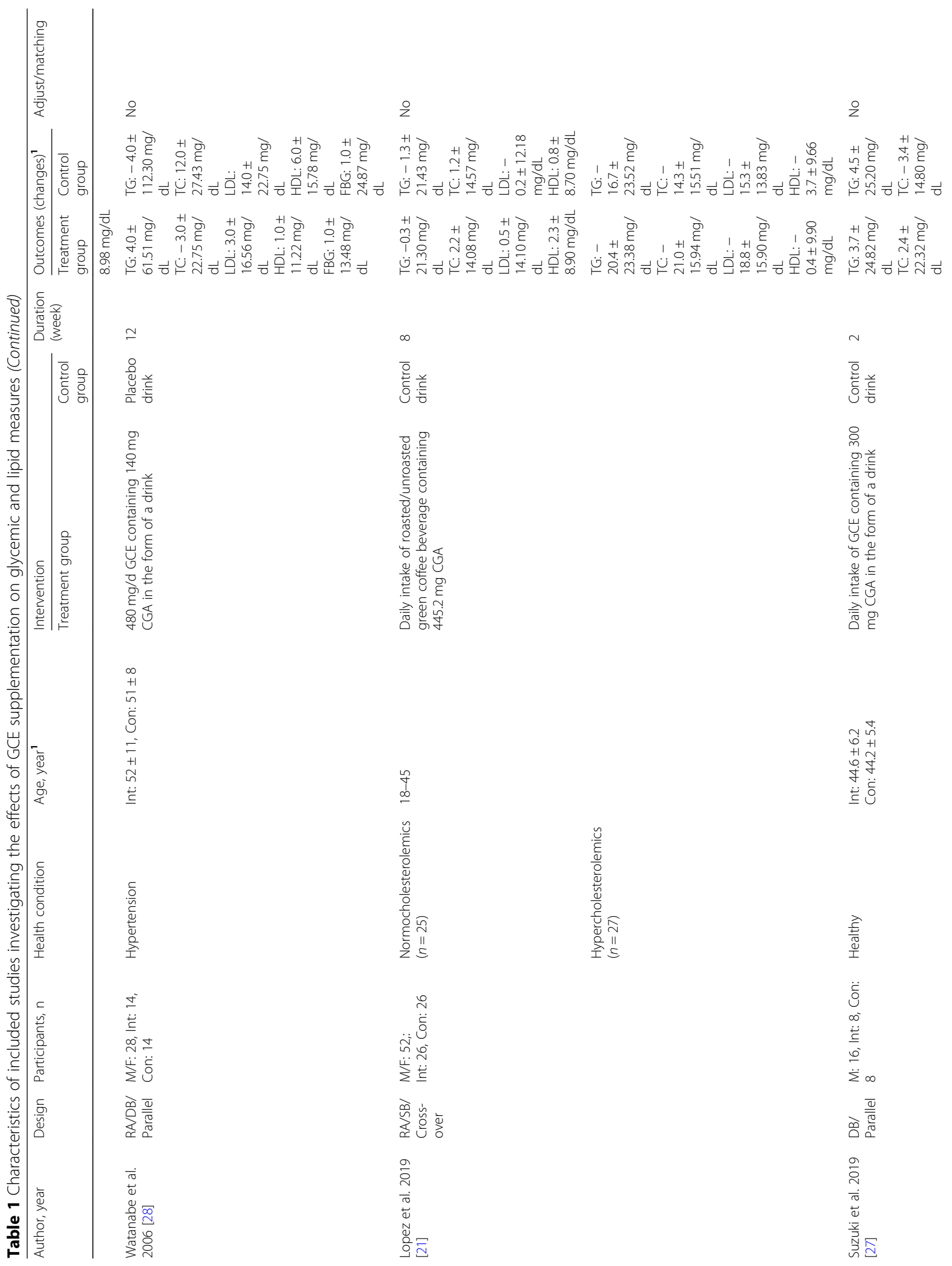




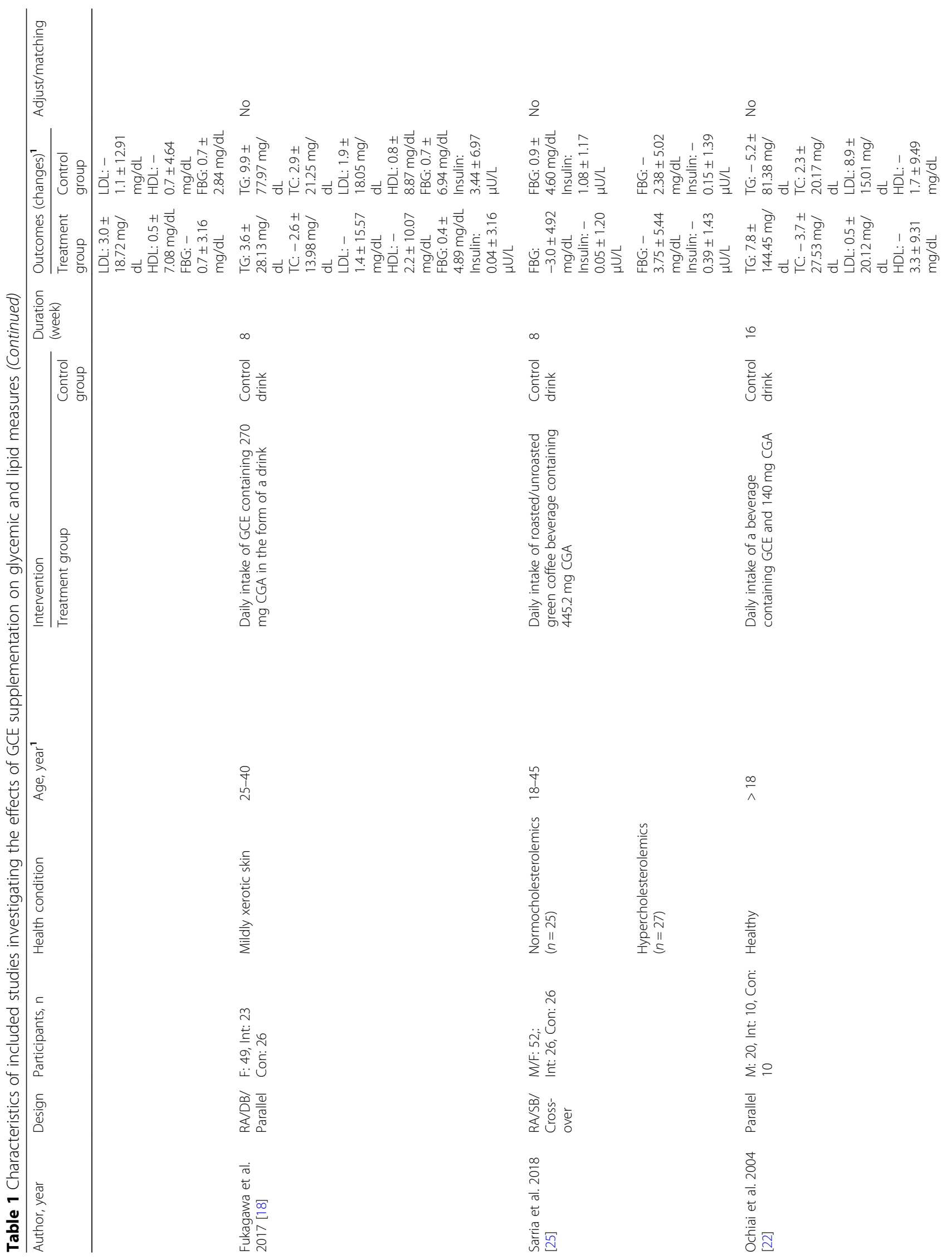




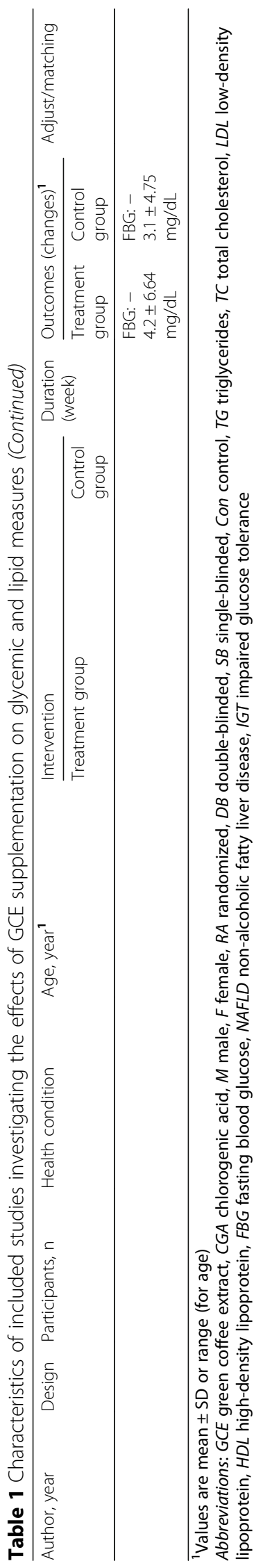


between 90 and $6000 \mathrm{mg} /$ day. Also, the dosage of CGA, as the main polyphenol in GCE, was between 13.5 and $1200 \mathrm{mg} /$ day. The duration of intervention varied from 2 to 16 weeks.

Of fourteen studies, six trials administered GCE in the form of supplement $[16,19,23,24,26,29]$, while eight studies administered in the form of powder added to boiled water or other beverages [17, 18, 20-22, 25, 27, 28]. Out of fourteen studies, two were performed on healthy individuals $[22,27]$ and others were conducted on subjects with overweight or obesity, dyslipidemia, hypertension, metabolic syndrome, non-alcoholic fatty liver disease, impaired glucose tolerance and mildly xerotic skin. According to the Cochrane Risk of Bias Assessment tool, none of the clinical trials had a low risk of bias in all domains of this tool (Supplemental Table 1).

Among eleven studies on FBG, four trials reported a lowering effect of GCE supplementation on FBG levels $[16,24-26,29]$ and the other trials revealed no significant effect. Only in two studies, GCE supplementation could reduce serum levels of insulin $[19,25]$ while the other trials could not find any significant change. There were 12 clinical trials on serum concentrations of TG, from which two studies revealed a lowering effect [26, 29], one study showed an increasing effect [17], and the others indicated no significant effect on serum TG concentrations following GCE supplementation. Out of thirteen studies on TC, 3 studies found a significant reduction in serum TC concentrations after supplementation with GCE $[19,26,29]$. Only one study revealed a reducing effect of GCE supplementation on serum concentrations of LDL [19] while others did not reach statistical significance. Among twelve studies on serum HDL levels, only two studies showed an increasing effect of GCE supplementation on serum concentrations of HDL $[17,19]$ whereas others failed to find any significant effect.

\section{Findings from the meta-analysis}

All fourteen clinical trials were included in the metaanalysis. These studies included 766 participants aged 18 years and over. There was a trial with three intervention arms with different dosages of GCE compared with one control group [20]. This study was considered as three separate studies. In two studies, findings were reported separately for normocholesterolemic and hypercholesterolemic participants $[21,25]$. Therefore, we considered each study as two separate studies.

\section{The effect of GCE on FBG levels}

Overall, 12 effect sizes from 11 clinical trials with a total sample size of 457 participants were included in the analysis [16, 18, 19, 22-29]. After combining effect sizes, we found a significant reducing effect of GCE supplementation on FBG levels (WMD: -2.35, 95\% CI: $-3.78,-0.92$ $\mathrm{mg} / \mathrm{dL}, P=0.001$ ) (Fig. 1). However, there was a moderate between-study heterogeneity $\left(I^{2}: 46.8, P=0.03\right)$. To detect the sources of between-study heterogeneity, we performed subgroup analyses according to gender (male, female, or both), length of intervention ( $\geq 8$ vs. $<8$ weeks), baseline levels of FBG ( $\geq 100$ vs. $<100 \mathrm{mg} / \mathrm{dL}$ ) and participants' compliance (acceptable, nonacceptable, or unclear) (Table 2). From these analyses, we found that gender, baseline levels of FBG and participants' compliance could explain between-study heterogeneity. In addition, GCE supplementation had a reducing effect on FBG in studies performed on both genders, those with an intervention duration of $\geq 8$ weeks, and those in which participants had a good adherence to the intervention. Findings from the sensitivity analysis revealed that the overall estimate did not depend on a particular study. Based on the Begg's test and visual inspection of funnel plot (Supplemental Figure $2 \mathrm{~A})$, no evidence of a publication bias was found $(P=$ 0.21 ).

In the non-linear dose-response analysis, we found that the association between dosage of CGA and FBG levels was in a non-linear fashion (Supplemental Figure $3 \mathrm{~A})$; such that greater reducing effect of CGA on FBG levels was seen at the dosage of $\geq 200 \mathrm{mg} /$ day $\left(\mathrm{P}_{\text {non-linear- }}\right.$ ity $=0.03)$.

\section{The effect of GCE on serum concentrations of insulin}

Combining 7 effect sizes from 6 studies [18, 19, 24-26, 29] that included 347 participants revealed that GCE supplementation resulted in a significant reduction in serum concentrations of insulin (WMD: -0.63, 95\% CI: $-1.11,-0.15 \mu \mathrm{U} / \mathrm{L}, P=0.01$ ) (Fig. 2). We observed a moderate between-study heterogeneity $\left(I^{2}: 49.4, P=\right.$ $0.06)$. In the subgroup analyses, we observed that between-study heterogeneity could be explained by gender and participants' compliance. A significant reducing effect of GCE supplementation on serum insulin concentrations was also seen in all subgroups. Based on the sensitivity analysis, no single study influenced the overall estimate. Also, no evidence of a substantial publication bias was found according to the Begg's test $(P=0.45)$ and funnel plot (Supplemental Figure 2B).

Based on the non-linear dose-response analysis, no significant effect of CGA dosage on serum insulin concentrations was seen $\left(P_{\text {non-linearity }}=0.62\right)$ (Supplemental Figure 3B).

\section{The effect of GCE on serum concentrations of TG}

Totally, 12 studies with a total sample size of 642 participants were included for this effect [17-24, 26-29]. Combining 15 effect sizes from these studies revealed no significant effect of GCE supplementation on serum TG concentrations (WMD: -3.17, 95\% CI: - 11.82, $5.49 \mathrm{mg} /$ 


\begin{tabular}{|c|c|c|}
\hline ID & WMD $(95 \%$ CI $)$ & Weight \\
\hline Ochiai et al. 2004 & $-1.10(-6.16,3.96)$ & 5.76 \\
\hline Watanabe et al. 2006 & $0.00(-14.82,14.82)$ & 0.89 \\
\hline Park et al. 2010 & $0.42(-3.83,4.67)$ & 7.30 \\
\hline Kim et al. 2012 & $-5.50(-9.03,-1.97)$ & 9.12 \\
\hline Haidari et al. 2017 & $-0.56(-2.29,1.17)$ & 15.73 \\
\hline Shahmohammadi et al. 2017 & $-4.55(-8.76,-0.34)$ & 7.40 \\
\hline Fukagawa et al. 2017 & $-0.30(-3.64,3.04)$ & 9.69 \\
\hline Roshan et al. 2018 & $-34.40(-65.09,-3.71)$ & 0.22 \\
\hline Zuniga et al. 2018 & $-5.41(-8.84,-1.98)$ & 9.40 \\
\hline Sarria et al. 2018 & $-3.90(-6.54,-1.26)$ & 12.05 \\
\hline Sarria et al. 2018 & $-1.37(-4.16,1.42)$ & 11.50 \\
\hline Suzuki et al. 2019 & $-1.40(-4.35,1.55)$ & 10.95 \\
\hline Overall $(\mathrm{I}$-squared $=46.8 \%, \mathrm{p}=0.037)$ & $-2.35(-3.78,-0.92)$ & 100.00 \\
\hline NOTE: Weights are from random effects analysis & & \\
\hline $\begin{array}{r} \\
-20\end{array}$ & $\begin{array}{l}1 \\
20\end{array}$ & \\
\hline
\end{tabular}

dL, $P=0.47$ ) (Fig. 3). However, between-study heterogeneity was significant $\left(I^{2}: 59.1, P=0.002\right)$. Subgroup analyses based on gender and length of follow-up could decrease the between-study heterogeneity. In addition, we found a significant lowering effect of GCE supplementation on serum concentrations of TG in studies performed on both genders. The sensitivity analysis revealed that the exclusion of any particular study did not change the overall estimate. We found no evidence of a substantial publication bias according to the Begg's test $(P=0.96)$ and funnel plot (Supplemental Figure 2C).

In the dose-response analysis, we found a significant non-linear association between CGA dosage and serum TG concentrations; such that dosage of CGA from low levels to $500 \mathrm{mg} /$ day had a significant lowering effect on TG levels $\left(P_{\text {non-linearity }}=0.01\right)$, while this beneficial effect was reduced from dosage of $500 \mathrm{mg} /$ day to higher amounts (Supplemental Figure 4A).
The effect of GCE on serum concentrations of TC

Overall, 16 effect sizes from 13 clinical trials [16-24, 26-29] with a sample size of 662 participants were included in the analysis. Combining these effect sizes, a significant reduction was seen in serum concentrations of TC following GCE supplementation (WMD: -4.51, 95\% CI: $-8.39,-0.64 \mathrm{mg} / \mathrm{dL}, P=0.02$ ) (Fig. 4). There was an evidence of moderate between-study heterogeneity $\left(I^{2}: 44.1, P=0.03\right)$. In the subgroup analyses, we found that gender, length of duration, baseline levels of TC and participants' compliance could explain betweenstudy heterogeneity. Based on these analyses, the effect of GCE supplementation on serum TC concentrations strengthened in studies performed on females and both genders, those with $\geq 8$ weeks' duration of follow-up and studies that were performed on participants with elevated baseline serum levels of TC $(\geq 200 \mathrm{mg} / \mathrm{dL})$. In the sensitivity analysis, significant association attenuated 
Table 2 Subgroup analysis on the effects of GCE supplementation on glycemic and lipid measures

\begin{tabular}{|c|c|c|c|c|c|c|}
\hline & \multirow[t]{2}{*}{ Effect size, $n$} & \multirow[t]{2}{*}{ WMD $^{1}$} & \multirow[t]{2}{*}{$95 \% \mathrm{Cl}^{1}$} & \multirow[t]{2}{*}{$P$-value ${ }^{2}$} & \multicolumn{2}{|c|}{ Heterogeneity } \\
\hline & & & & & $1^{2}(\%)^{3}$ & P-heterogeneity ${ }^{4}$ \\
\hline \multicolumn{7}{|l|}{ The effect of GCE on FBS } \\
\hline Overall effect size & 12 & -2.35 & $-3.78,-0.92$ & 0.001 & 45.8 & 0.037 \\
\hline \multicolumn{7}{|l|}{ Participants' gender } \\
\hline Male & 2 & -1.32 & $-3.87,1.22$ & 0.31 & 0.0 & 0.92 \\
\hline Female & 4 & -1.13 & $-2.47,0.21$ & 0.098 & 57.5 & 0.07 \\
\hline Both genders & 6 & -3.55 & $-5.10,-2.01$ & $<0.001$ & 36.3 & 0.17 \\
\hline \multicolumn{7}{|l|}{ Intervention duration (week) } \\
\hline$\geq 8$ & 11 & -2.13 & $-3.12,-1.13$ & $<0.001$ & 51.1 & 0.025 \\
\hline$<8$ & 1 & -1.40 & $-4.35,1.55$ & 0.35 & - & - \\
\hline \multicolumn{7}{|l|}{ Baseline FBS } \\
\hline Elevated ( $\geq 100$ mg/dL) & 3 & -5.28 & $-7.93,-2.63$ & $<0.001$ & 44.10 & 0.17 \\
\hline Normal $(<100$ mg/dL) & 9 & -1.59 & $-2.59,-0.58$ & 0.002 & 24.20 & 0.23 \\
\hline \multicolumn{7}{|l|}{ Compliance } \\
\hline Acceptable & 5 & -3.59 & $-5.15,-2.04$ & $<0.001$ & 47.6 & 0.11 \\
\hline Unacceptable/Unclear & 7 & -1.16 & $-2.34,0.02$ & 0.053 & 15.5 & 0.31 \\
\hline \multicolumn{7}{|c|}{ The effect of GCE on insulin levels } \\
\hline Overall effect size & 7 & -0.63 & $-1.11,-0.15$ & 0.01 & 49.4 & 0.07 \\
\hline \multicolumn{7}{|l|}{ Participants' gender } \\
\hline Female & 3 & -0.31 & $-0.52,-0.10$ & 0.004 & 54.4 & 0.11 \\
\hline Both genders & 4 & -0.77 & $-1.21,-0.33$ & 0.001 & 26.0 & 0.26 \\
\hline \multicolumn{7}{|l|}{ Compliance } \\
\hline Acceptable & 4 & -0.77 & $-1.21,-0.33$ & 0.001 & 26.0 & 0.26 \\
\hline Unacceptable/Unclear & 3 & -0.31 & $-0.52,-0.10$ & 0.004 & 54.4 & 0.11 \\
\hline \multicolumn{7}{|c|}{ The effect of GCE on TG levels } \\
\hline Overall effect size & 15 & -3.17 & $-11.82,5.49$ & 0.47 & 59.1 & 0.002 \\
\hline \multicolumn{7}{|l|}{ Participants' gender } \\
\hline Male & 5 & -1.05 & $-11.70,9.60$ & 0.85 & 0.0 & 0.99 \\
\hline Female & 4 & 2.76 & $-5.18,10.70$ & 0.49 & 79.6 & 0.002 \\
\hline Both genders & 6 & -7.83 & $-15.22,-0.44$ & 0.04 & 68.0 & 0.008 \\
\hline \multicolumn{7}{|l|}{ Intervention duration (week) } \\
\hline$\geq 8$ & 11 & -2.87 & $-8.27,2.53$ & 0.29 & 70.6 & $<0.001$ \\
\hline$<8$ & 4 & -1.21 & $-11.91,9.50$ & 0.83 & 0.0 & 0.99 \\
\hline \multicolumn{7}{|l|}{ Baseline TG } \\
\hline Elevated ( $\geq 150 \mathrm{mg} / \mathrm{dL}$ ) & 4 & -4.58 & $-12.77,3.60$ & 0.27 & 74.9 & 0.008 \\
\hline Normal (<150 mg/dL) & 11 & -1.44 & $-7.41,4.53$ & 0.64 & 54.4 & 0.02 \\
\hline \multicolumn{7}{|l|}{ Compliance } \\
\hline Acceptable & 8 & -6.14 & $-12.44,0.17$ & 0.06 & 57.3 & 0.02 \\
\hline Unacceptable/Unclear & 7 & 2.55 & $-4.93,10.03$ & 0.50 & 59.5 & 0.02 \\
\hline \multicolumn{7}{|c|}{ The effect of GCE on TC levels } \\
\hline Overall effect size & 16 & -4.51 & $-8.39,-0.64$ & 0.02 & 44.1 & 0.03 \\
\hline \multicolumn{7}{|l|}{ Participants' gender } \\
\hline Male & 5 & -1.51 & $-6.78,3.75$ & 0.57 & 5.6 & 0.38 \\
\hline Female & 5 & -5.29 & $-8.87,-1.71$ & 0.004 & 44.2 & 0.13 \\
\hline Both genders & 6 & -7.48 & $-12.11,-2.86$ & 0.002 & 60.4 & 0.03 \\
\hline \multicolumn{7}{|l|}{ Intervention duration (week) } \\
\hline$\geq 8$ & 12 & -6.11 & $-8.91,-3.30$ & $<0.001$ & 45.9 & 0.04 \\
\hline
\end{tabular}


Table 2 Subgroup analysis on the effects of GCE supplementation on glycemic and lipid measures (Continued)

\begin{tabular}{|c|c|c|c|c|c|c|}
\hline & \multirow[t]{2}{*}{ Effect size, $n$} & \multirow[t]{2}{*}{$W^{\prime} D^{1}$} & \multirow[t]{2}{*}{$95 \% \mathrm{Cl}^{1}$} & \multirow[t]{2}{*}{$P$-value ${ }^{2}$} & \multicolumn{2}{|c|}{ Heterogeneity } \\
\hline & & & & & $1^{2}(\%)^{3}$ & P-heterogeneity ${ }^{4}$ \\
\hline$<8$ & 4 & -1.22 & $-6.65,4.22$ & 0.66 & 26.0 & 0.26 \\
\hline \multicolumn{7}{|l|}{ Baseline TC } \\
\hline Elevated ( $\geq 200 \mathrm{mg} / \mathrm{dL}$ ) & 8 & -6.06 & $-9.01,-3.12$ & $<0.001$ & 26.2 & 0.22 \\
\hline Normal $(<200$ mg/dL) & 8 & -2.60 & $-7.28,2.09$ & 0.28 & 55.8 & 0.03 \\
\hline \multicolumn{7}{|l|}{ Compliance } \\
\hline Acceptable & 8 & -4.88 & $-8.53,-1.23$ & 0.009 & 59.4 & 0.02 \\
\hline Unacceptable/Unclear & 8 & -5.26 & $-8.66,-1.85$ & 0.003 & 27.0 & 0.21 \\
\hline \multicolumn{7}{|c|}{ The effect of GCE on LDL levels } \\
\hline Overall effect size & 15 & -2.02 & $-5.58,1.54$ & 0.27 & 47.6 & 0.02 \\
\hline \multicolumn{7}{|l|}{ Participants' gender } \\
\hline Male & 5 & 0.58 & $-4.59,5.76$ & 0.83 & 3.4 & 0.39 \\
\hline Female & 4 & -6.96 & $-10.01,-3.90$ & $<0.001$ & 61.1 & 0.05 \\
\hline Both genders & 6 & -1.44 & $-5.45,2.57$ & 0.48 & 25.5 & 0.24 \\
\hline \multicolumn{7}{|l|}{ Intervention duration (week) } \\
\hline$\geq 8$ & 11 & -5.01 & $-7.42,-2.61$ & $<0.001$ & 47.9 & 0.04 \\
\hline$<8$ & 4 & 1.70 & $-3.79,7.19$ & 0.54 & 0.0 & 0.44 \\
\hline \multicolumn{7}{|l|}{ Baseline LDL } \\
\hline Elevated ( $\geq 130 \mathrm{mg} / \mathrm{dL}$ ) & 2 & 0.10 & $-5.73,5.53$ & 0.97 & 28.9 & 0.24 \\
\hline Normal (<130 mg/dL) & 13 & -4.63 & $-7.02,-2.24$ & $<0.001$ & 48.3 & 0.03 \\
\hline \multicolumn{7}{|l|}{ Compliance } \\
\hline Acceptable & 8 & 0.01 & $-3.38,3.41$ & 0.99 & 11.1 & 0.34 \\
\hline Unacceptable/Unclear & 7 & -6.79 & $-9.68,-3.90$ & $<0.001$ & 39.4 & 0.13 \\
\hline \multicolumn{7}{|c|}{ The effect of GCE on HDL levels } \\
\hline Overall effect size & 15 & 1.08 & $-0.22,2.38$ & 0.10 & 37.6 & 0.07 \\
\hline \multicolumn{7}{|l|}{ Participants' gender } \\
\hline Male & 5 & 0.06 & $-2.16,2.27$ & 0.96 & 0.0 & 0.99 \\
\hline Female & 4 & 2.92 & $2.46,3.39$ & $<0.001$ & 62.0 & 0.05 \\
\hline Both genders & 6 & 0.63 & $-1.37,2.62$ & 0.54 & 0.0 & 0.59 \\
\hline \multicolumn{7}{|l|}{ Intervention duration (week) } \\
\hline$\geq 8$ & 11 & 2.79 & $2.34,3.24$ & $<0.001$ & 43.0 & 0.06 \\
\hline$<8$ & 4 & 0.18 & $-2.12,2.49$ & 0.88 & 0.0 & 0.99 \\
\hline \multicolumn{7}{|l|}{ Baseline HDL } \\
\hline Low $(<40$ mg/dL) & 5 & 2.87 & $2.41,3.33$ & $<0.001$ & 56.4 & 0.06 \\
\hline Normal ( $\geq 40$ mg/dL) & 10 & 0.34 & $-1.36,2.04$ & 0.69 & 0.0 & 0.81 \\
\hline \multicolumn{7}{|l|}{ Compliance } \\
\hline Acceptable & 8 & 0.51 & $-1.06,2.09$ & 0.52 & 0.0 & 0.91 \\
\hline Unacceptable/Unclear & 7 & 2.88 & $2.42,3.14$ & $<0.001$ & 48.7 & 0.07 \\
\hline
\end{tabular}

Obtained from the fixed-effects model

${ }^{2}$ Refers to the mean $(95 \% \mathrm{Cl})$

${ }^{3}$ Inconsistency, percentage of variation across studies due to heterogeneity

${ }^{4}$ Obtained from the Q-test

Abbreviations: GCE green coffee extract, WMD weighted mean difference, $C l$ confidence interval, $T G$ triglycerides, $T C$ total cholesterol, $L D L$ low-density

lipoprotein, $H D L$ high-density lipoprotein, FBG fasting blood glucose

after exclusion of the study by Heidari et al. [19] (WMD: $-4.11,95 \% \mathrm{CI}:-8.61,0.40 \mathrm{mg} / \mathrm{dL}, P=0.07)$ and the study by Shahmohammadi et al. [26] (WMD: $-3.69,95 \%$ CI: $-7.47,0.08 \mathrm{mg} / \mathrm{dL}, P=0.05)$. However, this effect was marginally significant. Visual inspection of funnel plot (Supplemental Figure 2D) and findings from the Begg's test revealed no evidence of a substantial publication bias $(P=0.58)$.

Based on the dose-response analysis, no significant non-linear association was observed between CGA 


\begin{tabular}{|c|c|c|c|}
\hline ID & & WMD $(95 \% \mathrm{CI})$ & Weight \\
\hline Park et al. 2010 & + & $0.24(-1.95,2.43)$ & 4.30 \\
\hline Haidari et al. 2017 & $i^{*}$ & $-0.30(-0.51,-0.09)$ & 35.09 \\
\hline Shahmohammadi et al. 2017 & 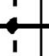 & $-0.72(-1.72,0.28)$ & 14.39 \\
\hline Fukagawa et al. 2017 & + & $-3.40(-6.38,-0.42)$ & 2.45 \\
\hline Roshan et al. 2018 & $\frac{1}{1}$ & $-2.43(-5.67,0.81)$ & 2.09 \\
\hline Sarria et al. 2018 & $\frac{1}{1}$ & $-1.13(-1.79,-0.47)$ & 22.12 \\
\hline Sarria et al. 2018 & to. & $-0.24(-1.00,0.52)$ & 19.57 \\
\hline Overall $(\mathrm{I}$-squared $=49.4 \%, \mathrm{p}=0.065)$ & 人i & $-0.63(-1.11,-0.15)$ & 100.00 \\
\hline NOTE: W eights are from random effects analysis & 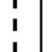 & & \\
\hline $\begin{array}{c}1 \\
-6.38\end{array}$ & 0 & $\begin{array}{c}1 \\
6.38\end{array}$ & \\
\hline
\end{tabular}

dosage and serum TC concentrations ( $\mathrm{P}_{\text {non-linearity }}=$ 0.15) (Supplemental Figure 4B).

\section{The effect of GCE on serum concentrations of $L D L$}

Considering 15 effect sizes from 12 studies [17-24, 26-29] which included 642 participants, no significant effect of GCE supplementation on serum concentrations of LDL was found (WMD: -2.02, 95\% CI: -5.58 , $1.54 \mathrm{mg} / \mathrm{dL}, P=0.26, I^{2}: 47.6, P=0.02$ ) (Fig. 5). Subgroup analyses based on gender, duration of followup, baseline values of LDL, and participants' compliance could decrease between-study heterogeneity. From these analyses, we found that GCE supplementation significantly reduced serum LDL concentrations in studies included only female subjects, studies with a follow-up duration of $\geq 8$ weeks, studies that included participants with normal baseline levels of LDL, and those with low or unclear compliance of participants. A sensitivity analysis revealed that the overall effect size was not influenced by a single study. No evidence of a substantial publication bias was seen based on the results from the Begg's test $(P=0.40)$ and funnel plot (Supplemental Figure 2E).

In the dose-response analysis, no significant association was found between CGA dosage and serum levels of LDL ( $\left.\mathrm{P}_{\text {non-linearity }}=0.27\right)$ (Supplemental Figure $\left.4 \mathrm{C}\right)$.

\section{The effect of GCE on serum concentrations of HDL}

Totally, 12 studies provided data on the effect of GCE supplementation on serum HDL concentrations [17-24, 26-29]. Combining 15 effect sizes from these studies revealed no significant effect of GCE supplementation on HDL levels (WMD: $1.08,95 \% \mathrm{CI}:-0.22,2.38 \mathrm{mg} / \mathrm{dL}$, $P=0.10$ ) (Fig. 6). A marginally significant between-study heterogeneity was seen in this regard $\left(I^{2}: 47.6, P=0.02\right)$. In the subgroup analyses, we found that gender, length of follow-up, baseline levels of HDL and participants' compliance were potential sources of heterogeneity. In addition, GCE supplementation resulted in a significant increase in serum HDL concentrations in trials included 


\begin{tabular}{|c|c|c|}
\hline ID & WMD $(95 \%$ CI $)$ & Weight \\
\hline Ochiai et al. 2004 & $-13.00(-89.77,115.77)$ & 0.67 \\
\hline Kozuma et al. 2005 & $-2.40(-24.71,19.91)$ & 7.16 \\
\hline Kozuma et al. 2005 & $1.50(-18.06,21.06)$ & 8.04 \\
\hline Kozuma et al. 2005 & $-3.40(-23.67,16.87)$ & 7.81 \\
\hline Watanabe et al. 2006 & $8.00(-59.08,75.08)$ & 1.48 \\
\hline Park et al. 2010 & $-16.52(-39.56,6.52)$ & 6.94 \\
\hline Haidari et al. 2017 & $1.00(-8.29,10.29)$ & 11.80 \\
\hline Shahmohammadi et al. 2017 & $-35.36(-55.02,-15.70)$ & 8.01 \\
\hline Fukagawa et al. 2017 & $-6.30(-38.40,25.80)$ & 4.74 \\
\hline Roshan et al. 2018 & $15.93(-23.45,55.31)$ & 3.57 \\
\hline Zuniga et al. 2018 & $-35.40(-62.73,-8.07)$ & 5.78 \\
\hline Aghaei et al. 2018 & $48.64(22.18,75.10)$ & 6.00 \\
\hline Lopez et al. 2019 & $1.00(-10.85,12.85)$ & 10.87 \\
\hline Lopez et al. 2019 & $-3.70(-16.21,8.81)$ & 10.62 \\
\hline Suzuki et al. 2019 & $-0.80(-25.32,23.72)$ & 6.51 \\
\hline Overall $(\mathrm{I}$-squared $=59.1 \%, \mathrm{p}=0.002)$ & $-3.17(-11.82,5.49)$ & 100.00 \\
\hline \multicolumn{3}{|l|}{ NOTE: Weights are from random effects analysis } \\
\hline $\begin{array}{c}1 \\
-116\end{array}$ & $\begin{array}{c}1 \\
116\end{array}$ & \\
\hline
\end{tabular}

only female subjects, studies with $\geq 8$ weeks of intervention duration, studies that were performed on participants with low levels of baseline serum HDL and studies with low or unclear adherence of participants to intervention. The sensitivity analysis revealed that the overall estimate depended on two studies $[18,26]$. When we excluded these studies, Shahmohammadi et al. (WMD: 1.32, 95\% CI: $0.03,2.62 \mathrm{mg} / \mathrm{dL}, P=0.04)$ and Fukagawa et al. studies (WMD: $1.44,95 \%$ CI: $0.23,2.64 \mathrm{mg} / \mathrm{dL}, P=$ 0.02 ), a significant increasing effect of GCE supplementation on serum HDL concentrations was observed. No evidence of a substantial publication bias was seen according to the Begg's test $(P=0.80)$ and funnel plot (Supplemental Figure 2F).

In the dose-response analysis, we found a significant non-linear association between CGA dosage and serum concentrations of HDL $\left(\mathrm{P}_{\text {non-linearity }}=0.01\right)$; such that from dosage of $100 \mathrm{mg} /$ day to higher levels, CGA administration had an increasing effect on serum HDL concentrations (Supplemental Figure 4D).

\section{Discussion}

The role of coffee consumption in the risk of cardiovascular diseases has been debated for many years. Recent meta-analyses of prospective studies showed no potential health risk associated with the coffee intake $[42,43]$ even when heavily consumed [44]. Also, an umbrella review of meta-analyses revealed the protective effects of coffee consumption against cardiovascular risk factors [45]. Unlike observational studies, considerable controversy exists among clinical trials. In the current study, we summarized evidence from clinical trials investigating the effect of GCE supplementation on lipid profile and some glycemic indices. We found that GCE supplementation significantly reduced FBG and insulin levels. Considering the lipid profile, GCE supplementation resulted 
ID

WMD $(95 \%$ CI $)$

Weight

Ochiai et al. 2004

Kozuma et al. 2005

Kozuma et al. 2005

Kozuma et al. 2005

Watanabe et al. 2006

Park et al. 2010

Kim et al. 2012

Haidari et al. 2017

Shahmohammadi et al. 2017

Fukagawa et al. 2017

Roshan et al. 2018

Zuniga et al. 2018

Aghaei et al. 2018

Lopez et al. 2019

Lopez et al. 2019

Suzuki et al. 2019

Overall $(\mathrm{I}$-squared $=44.1 \%, \mathrm{p}=0.030)$

NOTE: Weights are from random effects analysis

$-37.8$

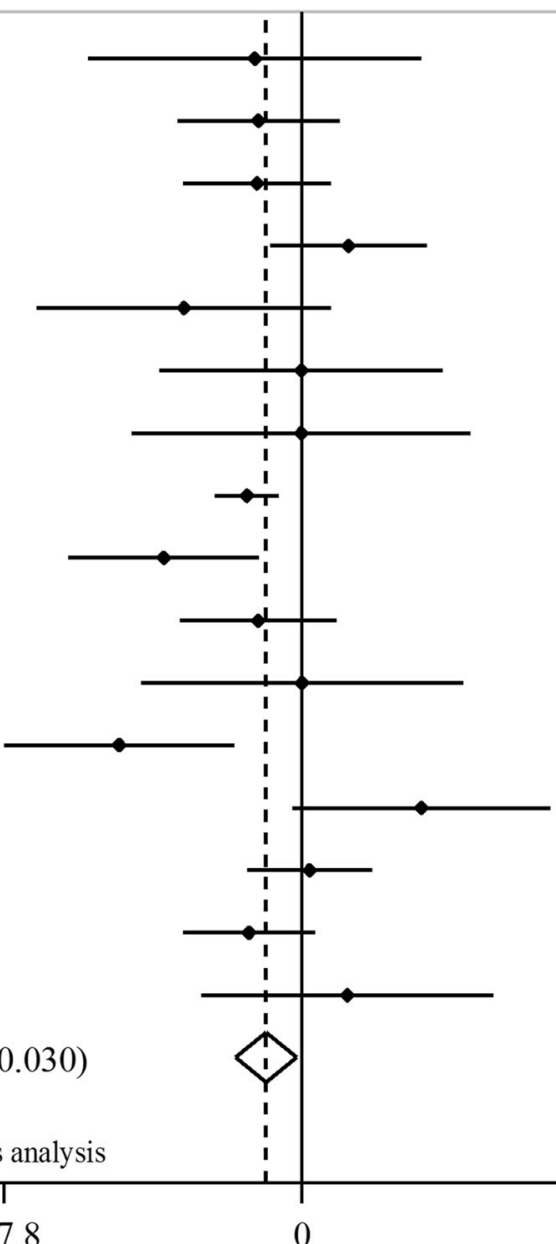

0
$-6.00(-27.16,15.16)$

2.79

$-5.50(-15.75,4.75)$

7.73

$-5.70(-15.07,3.67)$

8.48

$5.90(-4.08,15.88)$

7.95

$-15.00(-33.67,3.67)$

3.42

$-0.10(-18.13,17.93)$

3.62

$-0.10(-21.61,21.41)$

2.72

$-7.00(-11.12,-2.88)$

14.15

$-17.51(-29.62,-5.40)$

6.36

$-5.50(-15.47,4.47)$

7.96

$0.00(-20.46,20.46)$

2.95

$-23.17(-37.78,-8.56)$

4.96

$15.13(-1.26,31.52)$

4.19

$1.00(-6.94,8.94)$

9.86

$-6.70(-15.09,1.69)$

9.40

$5.80(-12.76,24.36)$

3.46

$-4.51(-8.39,-0.64)$

100.00

Fig. 4 Forest plot for the effect of GCE supplementation on serum TC concentrations, expressed as mean differences between intervention and control groups. Horizontal lines represent 95\% Cls. Diamonds represent pooled estimates from random-effects analysis. GCE: green coffee extract, TC: total cholesterol, WMD: weighted mean difference, Cl: confidence interval

in a significant decrease in TC concentrations, but results from other lipid measures were not significant. However, GCE supplementation improved serum levels of TG, LDL, and HDL in some subgroups of studies. Also, in the non-linear dose-response analyses, we found that the associations between CGA dosage and serum levels of FBS, TG, and HDL were in a non-linear fashion.

We found a significant reduction in both serum levels of FBG and insulin following GCE supplementation. In line with our findings, Morvaridi et al. reported a beneficial effect of green coffee consumption on glycemic indices and cardio-metabolic risk factors in adults [46]. In contrast with our findings, in a meta-analysis, Kondo et al. reported no significant effect of caffeinated/decaffeinated coffee consumption on FBS and insulin levels [47]. The observed controversy in this regard might be due to the use of different types of coffee across previous clinical trials. Green coffee contains a higher amount of CGA compared with other types of coffee. It seems that the anti-diabetic effect of green coffee is attributed to its CGA content. As seen in the dose-response analysis, the stronger reducing effect of GCE on FBG was observed at CGA dosage of $200 \mathrm{mg} / \mathrm{d}$ or more. CGA increases peripheral glucose disposal through activating AMPactivated protein kinase (AMPK) [48]. It also reduces glucose production by gluconeogenesis and glycogenolysis through inhibiting glucose-6-phosphatase [49]. Moreover, increased serum concentrations of adiponectin, a protein hormone released from adipose tissue that modulates glucose regulation and fatty acid oxidation, may play a role in the metabolic effect of CGA [50]. Reducing blood glucose through the mentioned pathways can also decrease insulin levels. 
ID

$\operatorname{WMD}(95 \% \mathrm{CI})$

Weight

Ochiai et al. 2004

Kozuma et al. 2005

Kozuma et al. 2005

Kozuma et al. 2005

Watanabe et al. 2006

Park et al. 2010

Haidari et al. 2017

Shahmohammadi et al. 2017

Fukagawa et al. 2017

Roshan et al. 2018

Zuniga et al. 2018

Aghaei et al. 2018

Lopez et al. 2019

Lopez et al. 2019

Suzuki et al. 2019

Overall (I-squared $=47.6 \%, p=0.021)$

NOTE: Weights are from random effects analysis

$$
-39.2
$$

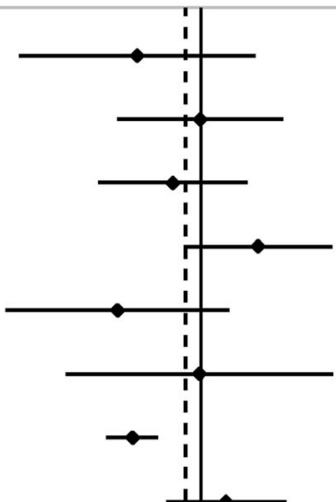

$-8.40(-23.96,7.16) \quad 3.98$

$-0.10(-11.02,10.82) \quad 6.46$

$-3.70(-13.56,6.16) \quad 7.29$

$7.50(-2.26,17.26) \quad 7.37$

$-11.00(-25.74,3.74) \quad 4.31$

$-0.21(-17.83,17.41) \quad 3.28$

$-9.00(-12.45,-5.55) \quad 14.29$

$3.31(-4.65,11.27) \quad 9.04$

$-3.30(-12.72,6.12) \quad 7.66$

$0.00(-14.64,14.64) \quad 4.35$

$-19.31(-39.20,0.58) \quad 2.68$

$5.56(-5.18,16.30) \quad 6.60$

$0.70(-6.61,8.01) \quad 9.74$

$-3.50(-11.45,4.45) \quad 9.05$

$4.10(-11.66,19.86) \quad 3.90$

$-2.02(-5.58,1.54) \quad 100.00$

Fig. 5 Forest plot for the effect of GCE supplementation on serum LDL concentrations, expressed as mean differences between intervention and control groups. Horizontal lines represent 95\% Cls. Diamonds represent pooled estimates from random-effects analysis. GCE: green coffee extract, LDL: low-density lipoprotein, WMD: weighted mean difference, Cl: confidence interval

In the overall meta-analysis of the effect of GCE on lipid profile, we found a reducing effect only on serum levels of TC. In agreement with our findings, a prospective cohort study indicated a significant inverse association between coffee consumption, particularly green coffee, and TC levels [51]. Also, in an experimental study, GCE administration could significantly reduce TC levels [52]. In contrast, available findings on the effects of other types of coffee on lipid profile are conflicting [30]. Different findings might be explained by different processing methods used for preparation of coffee. In a systematic review, Penson et al. reported that the type of coffee and the methods of preparation are important for the effect of coffee consumption on serum levels of lipoproteins [53]. In addition, different duration of intervention, recruiting participants with different health conditions and different quality of clinical trials are other reasons for the observed discrepancy across clinical trials investigating the effects of coffee consumption on TC levels.

Although the overall analysis did not show significant effects of GCE supplementation on serum levels of LDL and HDL, we found favorable effects in studies that recruited female subjects. Further, the TC-lowering effect increased in studies conducted on either gender or female subjects. The analysis also showed a significant reducing effect of GCE on serum levels of TG and FBG among studies that included both sexes. It seems that sex may mediate the effect of GCE on lipid profile with a stronger effect observed in women. It has been shown that CGA is more durable in women than men and therefore, had a longer effect on women than men [54]. Also, the sex differences may be mediated by changes in steroid hormone levels [55].

In our meta-analysis, the beneficial effects of GCE supplementation were mostly observed in RCTs with a long 


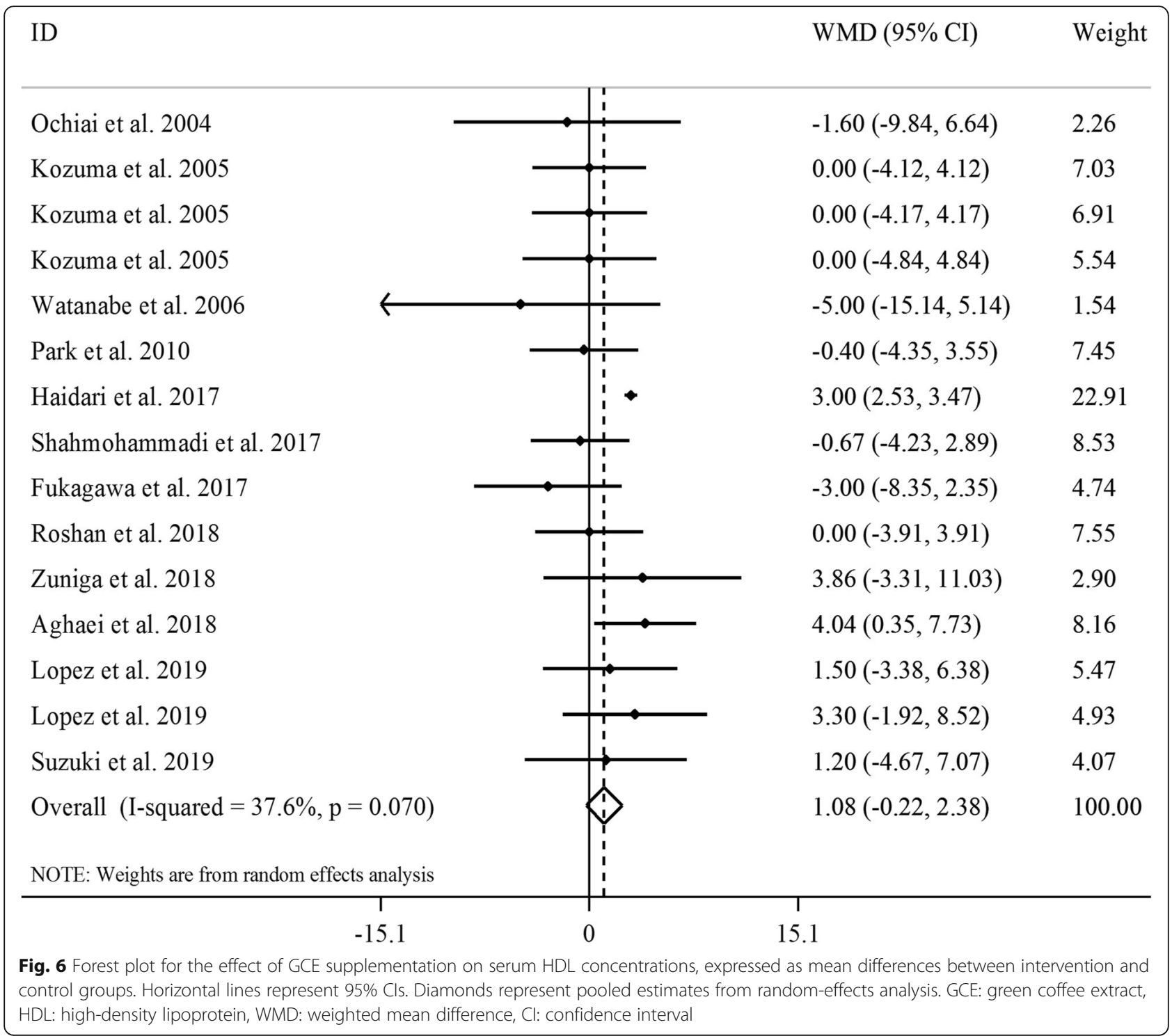

duration of the intervention ( $\geq 8$ weeks). It is consistent with the results from the previous meta-analysis on green coffee consumption in which a longer duration (> 8 weeks) was more effective on lipid profile [56]. In a network meta-analysis, Kondo et al. concluded that clinical trials with a longer duration of intervention can better clarify the potential effects of coffee [47]. Thus, green coffee consumption should be long enough to improve the lipid profile.

Dose-response analysis demonstrated a significant increment in serum levels of HDL from CGA dosage of 100 $\mathrm{mg} /$ day to higher amounts. Moreover, we found a significant non-linear association between CGA dosage and serum TG concentrations. However, the TG-lowering effect of CGA was decreased at a dosage of $500 \mathrm{mg} / \mathrm{d}$ and over. The effect of green coffee on lipid profile was also dose-dependent in earlier meta-analyses of RCTs $[56,57]$.
Therefore, the dosage of CGA is a potential moderator for the beneficial effect of GCE on lipid profile. CGA may exert its lipid-lowering effects through inhibition of the lipids absorption and the formation of cholesterol micelles. Also, CGA is involved in modifying hepatic metabolism of cholesterol and fatty acids by inhibiting pancreatic lipase and hydroxymethyl glutaryl Co-A reductase and increasing the activity of fatty acid beta-oxidation and the expression of peroxisome proliferator-activated receptor-alpha in the liver $[13,58]$. Based on our finding, however, higher dosages of more than $500 \mathrm{mg} / \mathrm{d}$ CGA is not recommended. Of note that higher intake of CGA may elevate homocysteine levels, a risk factor for cardiovascular disease, and stimulate the release of adrenaline generating several effects on the cardiovascular system including increased blood pressure and reduced insulin sensitivity $[50,59,60]$. 
The current meta-analysis was the first to summarize available findings on the effects of GCE supplementation on glycemic and lipid profiles. The selected studies were from different countries which increase the generalization of findings to the various ethnic groups. Other strengths of this meta-analysis were the inclusion of all clinical trials written in all languages, lack of publication bias, and moderate-to-high quality of the most included studies. However, some limitations should be considered when interpreting our findings. These limitations include the lack of evaluation of participants' compliance in a limited number of RCTs, the lack of controlling for baseline values of glycemic and lipid measures in some others, short duration of intervention in some trials, and different health conditions of participants across included studies. Also, we could not find the effect of GCE supplementation on other glycemic indices such as HbA1c due to limited number of studies.

\section{Conclusion}

GCE supplementation had favorable effects on glycemic indices including FBG and insulin levels. In terms of lipid profile, GCE supplementation led to a significant reduction in serum $\mathrm{TC}$, particularly in individuals with elevated levels of TC. This suggests GCE as a promising antihyperlipidemic agent since some patients do not achieve cholesterol reduction goals or cannot tolerate statins due to adverse effects [61]. We also found a significant favorable effect of GCE on serum levels of TG, LDL, and HDL in some subgroups. The effect was more prominent in women and studies with a long duration of intervention. Further studies are required to find the effect of GCE supplementation on patient-reported outcomes including quality of life, as well as the effect of genetic polymorphisms on the pharmacokinetics of CGA to explain the interindividual variability.

\section{Supplementary information}

Supplementary information accompanies this paper at https://doi.org/10. 1186/s12937-020-00587-z.

Additional file 1: Supplemental Table 1. Results of risk of bias assessment for clinical trials included in the current meta-analysis on the effects of GCE supplementation on glycemic and lipid measures'. Supplemental Figure 1. Flow diagram of study selection. Supplemental Figure 2. Funnel plots for the effect of GCE supplementation on serum levels of FBG (A), insulin (B), TG (C), TC (D), LDL (E), and HDL (F). WMD: weighted mean difference, FBG: fasting blood glucose, TG: triglyceride, LDL: low-density lipoprotein, HDL: high-density lipoprotein. Supplemental Figure 3. Non-linear dose-response effects of CGA dosage $(\mathrm{mg} / \mathrm{d})$ on (A) FBG and (B) serum levels of insulin. The $95 \% \mathrm{Cl}$ is demonstrated in the shaded regions. CGA: chlorogenic acid, FBG: fasting blood glucose. Supplemental Figure4. Non-linear dose-response effects of CGA dosage $(\mathrm{mg} / \mathrm{d})$ on serum concentrations of (A) TG, (B) TC, (C) LDL, and (D) HDL. The $95 \% \mathrm{Cl}$ is demonstrated in the shaded regions. CGA: chlorogenic acid, TG: triglycerides, TC: total cholesterol, LDL: low-density lipoprotein, HDL: high-density lipoprotein

\section{Acknowledgements}

Not applicable.

\section{Authors' contributions}

$\mathrm{OA}, \mathrm{MS}, \mathrm{MN}$ and OS contributed in systematic search and data extraction. MK, AP, AS and OS contributed in statistical analyses and data interpretation. $\mathrm{OS}, \mathrm{BP}$ and MS contributes in manuscript drafting and data interpretation. All authors approved the final manuscript for submission.

\section{Funding}

This study was supported by the Gerash University of Medical Sciences, Gerash, Iran

\section{Availability of data and materials}

The datasets analyzed during the current study are available from the corresponding author on reasonable request.

Ethics approval and consent to participate

Not applicable.

Consent for publication

Not applicable.

\section{Competing interests}

The authors declare that they have no competing interests.

\section{Author details}

${ }^{1}$ Nutritional Health Research Center, Lorestan University of Medical Sciences, Khorramabad, Iran. ${ }^{2}$ Department of Nutrition, School of Allied Medical Sciences, Ahvaz Jundishapur University of Medical Sciences, Ahvaz, Iran. ${ }^{3}$ Student Research Committee, Shiraz University of Medical Sciences, Shiraz, Iran. ${ }^{4}$ Department of Operating Room Nursing, School of Nursing and Midwifery, Shiraz University of Medical Sciences, Shiraz, Iran. ${ }^{5}$ Department of Epidemiology, School of Public Health, Shahid Beheshti University of Medical Sciences, Tehran, Iran. ${ }^{6}$ Department of Epidemiology, School of Public Health, Iran University of Medical Sciences, Tehran, Iran. ${ }^{7}$ Social Determinants of Health Research Center, Research Institute for Health Development, Kurdistan University of Medical Sciences, Sanandaj, Iran. ${ }^{8}$ Department of Nutrition, School of Health, Yasuj University of Medical Sciences, Yasuj, Iran. ${ }^{9}$ Gerash University of Medical Sciences, Gerash, Iran. ${ }^{10}$ Students' Scientific Research Center, Tehran University of Medical Sciences, Tehran, Iran. "1Department of Community Nutrition, School of Nutritional Sciences and Dietetics, Tehran University of Medical Sciences, Tehran, Iran.

Received: 27 January 2020 Accepted: 7 July 2020

Published online: 14 July 2020

References

1. Leong DP, Joseph PG, McKee M, Anand SS, Teo KK, Schwalm JD, et al. Reducing the global burden of cardiovascular disease, part 2: prevention and treatment of cardiovascular disease. Circ Res. 2017;121:695-710..

2. Pol T, Held C, Westerbergh J, Lindback J, Alexander JH, Alings M, et al. Dyslipidemia and risk of cardiovascular events in patients with atrial fibrillation treated with Oral anticoagulation therapy: insights from the ARIS TOTLE (Apixaban for reduction in stroke and other thromboembolic events in atrial fibrillation) trial. J Am Heart Assoc. 2018;7.

3. Abdul-Ghani M, DeFronzo RA, Jayyousi A. Prediabetes and risk of diabetes and associated complications: impaired fasting glucose versus impaired glucose tolerance: does it matter? Curr Opin Clin Nutr Metab Care. 2016;19: 394-9.

4. Katan MB, Grundy SM, Jones P, Law M, Miettinen T, Paoletti R. Efficacy and safety of plant stanols and sterols in the management of blood cholesterol levels. Mayo Clin Proc. 2003;78:965-78.

5. Anderson JW, Konz EC. Obesity and disease management: effects of weight loss on comorbid conditions. Obes Res. 2001:9(Suppl 4):326s-34s.

6. Ginsberg HN. Is hypertriglyceridemia a risk factor for atherosclerotic cardiovascular disease? A simple question with a complicated answer. Ann Intern Med. 1997;126:912-4.

7. Saaristo T, Moilanen L, Korpi-Hyovalti E, Vanhala M, Saltevo J, Niskanen L, et al. Lifestyle intervention for prevention of type 2 diabetes in primary 
health care: one-year follow-up of the Finnish National Diabetes Prevention Program (FIN-D2D). Diabetes Care. 2010;33:2146-51.

8. DiNicolantonio JJ, Harcombe Z, O'Keefe JH. Problems with the 2015 dietary guidelines for Americans: an alternative. Mo Med. 2016;113:93-7.

9. Butt MS, Sultan MT. Coffee and its consumption: benefits and risks. Crit Rev Food Sci Nutr. 2011:51:363-73.

10. Buscemi S, Verga S, Batsis JA, Donatelli M, Tranchina MR, Belmonte $S$, et al. Acute effects of coffee on endothelial function in healthy subjects. Eur J Clin Nutr. 2010;64:483-9.

11. Upadhyay R, Mohan Rao LJ. An outlook on chlorogenic acids-occurrence, chemistry, technology, and biological activities. Crit Rev Food Sci Nutr. 2013; 53:968-84.

12. Tajik N, Tajik M, Mack I, Enck P. The potential effects of chlorogenic acid, the main phenolic components in coffee, on health: a comprehensive review of the literature. Eur J Nutr. 2017;56:2215-44.

13. Cho AS, Jeon SM, Kim MJ, Yeo J, Seo KI, Choi MS, et al. Chlorogenic acid exhibits anti-obesity property and improves lipid metabolism in high-fat diet-induced-obese mice. Food Chem Toxicol. 2010;48:937-43.

14. Ong KW, Hsu A, Tan BK. Anti-diabetic and anti-lipidemic effects of chlorogenic acid are mediated by ampk activation. Biochem Pharmacol. 2013;85:1341-51.

15. Jin S, Chang C, Zhang L, Liu Y, Huang X, Chen Z. Chlorogenic acid improves late diabetes through adiponectin receptor signaling pathways in $\mathrm{db} / \mathrm{db}$ mice. PLoS One. 2015;10:e0120842 e.

16. Amirian ME, Fazilat-Pour M. Simple and multivariate relationships between spiritual intelligence with general health and happiness. J Relig Health. 2016;55:1275-88.

17. Aghaei F, Shadmehri S, Pirbeyg DZ. The effect of aerobic training with green coffee on body composition and lipid profile in overweight women \% report of. Health Care. 2018;4:38-46.

18. Fukagawa S, Haramizu S, Sasaoka S, Yasuda Y, Tsujimura H, Murase T. Coffee polyphenols extracted from green coffee beans improve skin properties and microcirculatory function. Biosci Biotechnol Biochem. 2017;81:1814-22.

19. Haidari F, Samadi M, Mohammadshahi M, Jalali MT, Engali KA. Energy restriction combined with green coffee bean extract affects serum adipocytokines and the body composition in obese women. Asia Pac J Clin Nutr. 2017;26:1048-54.

20. Kozuma K, Tsuchiya S, Kohori J, Hase T, Tokimitsu I. Antihypertensive effect of green coffee bean extract on mildly hypertensive subjects. Hypertens Res. 2005;28:711-8.

21. Martinez-Lopez S, Sarria B, Mateos R, Bravo-Clemente L. Moderate consumption of a soluble green/roasted coffee rich in caffeoylquinic acids reduces cardiovascular risk markers: results from a randomized, cross-over, controlled trial in healthy and hypercholesterolemic subjects. Eur J Nutr. 2019;58:865-78.

22. Ochiai R, Jokura H, Suzuki A, Tokimitsu I, Ohishi M, Komai N, et al. Green coffee bean extract improves human vasoreactivity. Hypertens Res. 2004;27: 731-7.

23. Park JY, Kim JY, Lee SP, Lee JH. The effect of green coffee bean extract supplementation on body fat reduction in overweight/obese women. Korean J Nutr. 2010;43:374-81.

24. Roshan H, Nikpayam O, Sedaghat M, Sohrab G. Effects of green coffee extract supplementation on anthropometric indices, glycaemic control, blood pressure, lipid profile, insulin resistance and appetite in patients with the metabolic syndrome: a randomised clinical trial. Br J Nutr. 2018;119:250-8.

25. Sarria B, Martinez-Lopez S, Sierra-Cinos JL, Garcia-Diz L, Mateos R, BravoClemente L. Regularly consuming a green/roasted coffee blend reduces the risk of metabolic syndrome. Eur J Nutr. 2018;57:269-78.

26. Shahmohammadi HA, Hosseini SA, Hajiani E, Malehi AS, Alipour M. Effects of green coffee bean extract supplementation on patients with non-alcoholic fatty liver disease: a randomized clinical trial. Hepat Mon. 2017;17(4):e12299.

27. Suzuki A, Nomura T, Jokura H, Kitamura N, Saiki A, Fujii A. Chlorogenic acidenriched green coffee bean extract affects arterial stiffness assessed by the cardio-ankle vascular index in healthy men: a pilot study. Int J Food Sci Nutr. 2019:1-7.

28. Watanabe T, Arai Y, Mitsui Y, Kusaura T, Okawa W, Kajihara Y, et al. The blood pressure-lowering effect and safety of chlorogenic acid from green coffee bean extract in essential hypertension. Clin Exp Hypertens. 2006;28: 439-49.

29. Zuniga LY, Aceves-de la Mora MCA, Gonzalez-Ortiz M, Ramos-Nunez JL, Martinez-Abundis E. Effect of Chlorogenic acid administration on glycemic control, insulin secretion, and insulin sensitivity in patients with impaired glucose tolerance. J Med Food. 2018;21:469-73.

30. Cai L, Ma D, Zhang Y, Liu Z, Wang P. The effect of coffee consumption on serum lipids: a meta-analysis of randomized controlled trials. Eur J Clin Nutr. 2012;66:872-7

31. Moher D, Liberati A, Tetzlaff J, Altman DG. Preferred reporting items for systematic reviews and meta-analyses: the PRISMA statement. Ann Intern Med. 2009;151:264-9.

32. Higgins JP, Altman DG, Gotzsche PC, Juni P, Moher D, Oxman AD, et al. The Cochrane Collaboration's tool for assessing risk of bias in randomised trials. Bmj. 2011;343:d5928.

33. Banth $\mathrm{S}$, Talwar C. Anasakti, the Hindu ideal, and its relationship to wellbeing and orientations to happiness. J Relig Health. 2012;51:934-46.

34. Hozo SP, Djulbegovic B, Hozo I. Estimating the mean and variance from the median, range, and the size of a sample. BMC Med Res Methodol. 2005:5:13.

35. Sadeghi O, Sadeghian M, Rahmani S, Maleki V, Larijani B, Esmaillzadeh A. Whole-Grain Consumption Does Not Affect Obesity Measures: An Updated Systematic Review and Meta-analysis of Randomized Clinical Trials. Adv Nutr. 2020;11(2):280-92.

36. Brondani LA, Assmann TS, de Souza BM, Boucas AP, Canani LH, Crispim D. Meta-analysis reveals the association of common variants in the uncoupling protein (UCP) 1-3 genes with body mass index variability. PLoS One. 2014;9: e96411.

37. Zahedi H, Djalalinia S, Sadeghi O, Asayesh H, Noroozi M, Gorabi AM, et al. Dietary inflammatory potential score and risk of breast Cancer: systematic review and meta-analysis. Clin Breast Cancer. 2018;18:e561-e70.

38. Wong RH, Garg ML, Wood LG, Howe PR. Antihypertensive potential of combined extracts of olive leaf, green coffee bean and beetroot: a randomized, double-blind, placebo-controlled crossover trial. Nutrients. 2014;6:4881-94.

39. Salamat S, Sharif SS, Nazary-Vanani A, Kord-Varkaneh H, Clark CC, Mohammadshahi M. The effect of green coffee extract supplementation on serum oxidized LDL cholesterol and total antioxidant capacity in patients with dyslipidemia: a randomized, double-blind, placebo-controlled trial. Eur J Integr Med. 2019;28:109-13.

40. Lecoultre V, Carrel G, Egli L, Binnert C, Boss A, MacMillan EL, et al. Coffee consumption attenuates short-term fructose-induced liver insulin resistance in healthy men. Am J Clin Nutr. 2014;99:268-75.

41. Soga S, Ota N, Shimotoyodome A. Stimulation of postprandial fat utilization in healthy humans by daily consumption of chlorogenic acids. Biosci Biotechnol Biochem. 2013;77:1633-6.

42. Crippa A, Discacciati A, Larsson SC, Wolk A, Orsini N. Coffee consumption and mortality from all causes, cardiovascular disease, and cancer: a doseresponse meta-analysis. Am J Epidemiol. 2014;180:763-75.

43. Coffee consumption and health: umbrella review of meta-analyses of multiple health outcomes. Bmj. 2018;360:k194.

44. Ding M, Bhupathiraju SN, Satija A, van Dam RM, Hu FB. Long-term coffee consumption and risk of cardiovascular disease: a systematic review and a dose-response meta-analysis of prospective cohort studies. Circulation. 2014; 129:643-59.

45. Poole R, Kennedy OJ, Roderick P, Fallowfield JA, Hayes PC, Parkes J. Coffee consumption and health: umbrella review of meta-analyses of multiple health outcomes. Bmj. 2017;359:15024.

46. Morvaridi M, Rayyani E, Jaafari M, Khiabani A, Rahimlou M. The effect of green coffee extract supplementation on cardio metabolic risk factors: a systematic review and meta-analysis of randomized controlled trials. J Diabetes Metab Disord. 2020;19:645-60.

47. Kondo Y, Goto A, Noma H, Iso H, Hayashi K, Noda M. Effects of coffee and tea consumption on glucose metabolism: a systematic review and network meta-analysis. Nutrients. 2018;11:48.

48. Ong KW, Hsu A, Tan BK. Chlorogenic acid stimulates glucose transport in skeletal muscle via AMPK activation: a contributor to the beneficial effects of coffee on diabetes. PLoS One. 2012;7:e32718.

49. Henry-Vitrac C, Ibarra A, Roller M, Merillon JM, Vitrac X. Contribution of chlorogenic acids to the inhibition of human hepatic glucose-6phosphatase activity in vitro by Svetol, a standardized decaffeinated green coffee extract. J Agric Food Chem. 2010;58:4141-4.

50. Wikoff D, Welsh BT, Henderson R, Brorby GP, Britt J, Myers E, et al. Systematic review of the potential adverse effects of caffeine consumption in healthy adults, pregnant women, adolescents, and children. Food Chem Toxicol. 2017;109:585-648. 
51. Nordestgaard AT, Nordestgaard BG. Coffee intake, cardiovascular disease and all-cause mortality: observational and Mendelian randomization analyses in 95 000-223 000 individuals. Int J Epidemiol. 2016;45:1938-52.

52. Ilmiawati C, Fitri F, Rofinda ZD, Reza M. Green coffee extract modifies body weight, serum lipids and TNF-alpha in high-fat diet-induced obese rats. BMC Res Notes. 2020;13:208.

53. Penson P, Serban MC, Ursoniu S, Banach M. Does coffee consumption alter plasma lipoprotein(a) concentrations? A systematic review. Crit Rev Food Sci Nutr. 2018;58:1706-14

54. Nybakken L, Horkka R, Julkunen-Tiitto R. Combined enhancements of temperature and UVB influence growth and phenolics in clones of the sexually dimorphic Salix myrsinifolia. Physiol Plant. 2012;145:551-64.

55. Temple JL, Bulkley AM, Briatico L, Dewey AM. Sex differences in reinforcing value of caffeinated beverages in adolescents. Behav Pharmacol. 2009;20: 731-41.

56. Ding F, Ma B, Nazary-Vannani A, Kord-Varkaneh H, Fatahi S, Papageorgiou $M$, et al. The effects of green coffee bean extract supplementation on lipid profile in humans: a systematic review and meta-analysis of randomized controlled trials. Nutr Metab Cardiovasc Dis. 2020;30:1-10.

57. Jee SH, He J, Appel LJ, Whelton PK, Suh I, Klag MJ. Coffee consumption and serum lipids: a meta-analysis of randomized controlled clinical trials. Am J Epidemiol. 2001;153:353-62.

58. Meng S, Cao J, Feng Q, Peng J, Hu Y. Roles of chlorogenic acid on regulating glucose and lipids metabolism: a review. Evid Based Complement Alternat Med. 2013;2013:801457.

59. Turnbull D, Rodricks JV, Mariano GF, Chowdhury F. Caffeine and cardiovascular health. Regul Toxicol Pharmacol. 2017;89:165-85.

60. Olthof MR, Hollman PC, Zock PL, Katan MB. Consumption of high doses of chlorogenic acid, present in coffee, or of black tea increases plasma total homocysteine concentrations in humans. Am J Clin Nutr. 2001;73:532-8.

61. Stein R, Ferrari F, Scolari F. Genetics, dyslipidemia, and cardiovascular disease: new insights. Curr Cardiol Rep. 2019;21:68.

\section{Publisher's Note}

Springer Nature remains neutral with regard to jurisdictional claims in published maps and institutional affiliations.

Ready to submit your research? Choose BMC and benefit from:

- fast, convenient online submission

- thorough peer review by experienced researchers in your field

- rapid publication on acceptance

- support for research data, including large and complex data types

- gold Open Access which fosters wider collaboration and increased citations

- maximum visibility for your research: over $100 \mathrm{M}$ website views per year

At $\mathrm{BMC}$, research is always in progress.

Learn more biomedcentral.com/submissions 